\title{
Effect of Red Light-Emitting Diodes Irradiation on Hemoglobin for Potential Hypertension Treatment Based on Confocal Micro-Raman Spectroscopy
}

\author{
Xuejun Qiu, ${ }^{1}$ Hanchuan Huang, 1 Zhitong Huang, ${ }^{2}$ Zhengfei Zhuang, ${ }^{1}$ \\ Zhouyi Guo, ${ }^{1}$ and Songhao Liu ${ }^{1}$ \\ ${ }^{1}$ MOE Key Laboratory of Laser Life Science \& SATCM Third Grade Laboratory of Chinese Medicine and Photonics Technology, \\ College of Biophotonics, South China Normal University, Guangzhou, China \\ ${ }^{2}$ Guzhen Productivity Promotion Center, Zhongshan, China
}

Correspondence should be addressed to Zhengfei Zhuang; zhuangzf@scnu.edu.cn and Zhouyi Guo; ann@scnu.edu.cn

Received 19 July 2016; Revised 17 October 2016; Accepted 23 October 2016; Published 11 January 2017

Academic Editor: Alessio Morelli

Copyright $\odot 2017$ Xuejun Qiu et al. This is an open access article distributed under the Creative Commons Attribution License, which permits unrestricted use, distribution, and reproduction in any medium, provided the original work is properly cited.

Red light-emitting diodes (LED) were used to irradiate the isolated hypertension hemoglobin (Hb) and Raman spectra difference was recorded using confocal micro-Raman spectroscopy. Differences were observed between the controlled and irradiated Hb by comparing the spectra records. The Raman spectrum at the $1399 \mathrm{~cm}^{-1}$ band decreased following prolonged LED irradiation. The intensity of the $1639 \mathrm{~cm}^{-1}$ band decreased dramatically in the first five minutes and then gradually increased in a time-dependent manner. This observation indicated that LED irradiation increased the ability of oxygen binding in $\mathrm{Hb}$. The appearance of the heme aggregation band at $1399 \mathrm{~cm}^{-1}$, in addition to the oxygen marker band at $1639 \mathrm{~cm}^{-1}$, indicated that, in our study, 30 min of irradiation with $15.0 \mathrm{~mW}$ was suitable for inhibiting heme aggregation and enhancing the oxygen-carrying capacity of Hb. Principal component analysis showed a one-to-one relationship between irradiated $\mathrm{Hb}$ at different time points and the corresponding Raman spectra. Our approach could be used to analyze the hemoglobin from patients with confocal micro-Raman spectroscopy and is helpful for developing new nondrug hypertension therapy.

\section{Introduction}

Hypertension is a global public-health challenge [1]. According to the report of the World Health Organization (WHO) in 2012 [2], one-third of the adult population is affected by this noncommunicable disease. Since hypertension is highly prevalent, it presents a major risk factor for many common underlying causes of diseases, including cardiovascular (CVD) $[3,4]$, stroke $[5,6]$, and kidney disease $[7$, 8]. Clinical manifestation of hypertension includes elevated arterial blood pressure and a weakened capacity for oxygen transport by erythrocytes, which would lead to the above listed diseases.

Common methods of treating hypertension include drug intervention $[9,10]$ and nondrug therapy $[11,12]$. Benefits of drug intervention on hypertension have been well documented, especially in high-risk individuals. However, treated patients that are affected by hypertension still have higher rates of hypertension-related CVD complications. This anomaly might result from the failure to radically cure hypertension, residual target organ damage such as left ventricular hypertrophy (LVH), or both [4]. Thus, drug intervention is not an optimal choice for hypertension treatment, and the disease pathogenesis of hypertension remains largely unknown [13]. Understanding the underlying molecular mechanisms of hypertension has aroused increasingly interests from clinicians and scientists.

Erythrocyte plays a crucial role in the human body for their prolific ability to transport oxygen and carbon dioxide to different tissues. The most important component in erythrocytes is $\mathrm{Hb}$, which is the primary oxygen-transport protein in humans. However, limited efforts have been applied to determine whether the $\mathrm{Hb}$ structure could be changed at the molecular level in erythrocytes of the hypertensive patient. 
Previous studies have found a positive association between $\mathrm{Hb}$ levels and the relative risk of hypertension [14]. Anand et al. [15] and Lipšic et al. [16] used erythropoietin treatment to recover the levels of $\mathrm{Hb}$, which appeared to improve patient outcome in the settings of chronic heart failure or kidney disease. Thus, identifying changes in the molecular structure of $\mathrm{Hb}$ in hypertension and recovering it could be potentially helpful for treating hypertension-related disease. However, as mentioned above, drug intervention in hypertensionassociated diseases is limited. Therefore, we have attempted to seek a novel nondrug therapy to recover the normal functional ability of $\mathrm{Hb}$ in transporting molecular oxygen.

There are several reports documenting significant positive effects of low-intensity laser therapy (LILT) on biological systems [17-20]. In recent in vitro studies on cell culture models, data was provided demonstrating the effects of lowlevel lasers in muscle, bone, skin, and certain tumors and in sperm and the biostimulatory effects on organisms at the molecular level [21]. The most commonly used laser in many LILT studies is the Helium-Neon (He-Ne, $\lambda=632.8 \mathrm{~nm}$ ) laser. In this study, we utilized red LED as a replacement of reduced low-intensity laser to irradiate erythrocytes in hypertension patients in vitro. LED typically consume low levels of power and are essentially heat-free. As a relatively mature technology, LED have been widely used in clinical treatment [22-25], and results have shown promising effects in these diseases. To our best knowledge, there are only few reports describing LED therapy on hypertension. In this study, after irradiating hypertension erythrocytes in vitro, we expected an inhibition of heme aggregation, a reduction of the metHb content, and an increase of the oxygen-carrying capacity of $\mathrm{Hb}$. In order to analyze the molecular changes of $\mathrm{Hb}$ after irradiation, a robust tool was urgently needed to characterize the levels of $\mathrm{Hb}$ and to provide new information of hypertension treatment at the molecular level.

Confocal micro-Raman spectroscopy is a potent tool in biomedical diagnostics due to its high sensitivity, increased capacity to be multiplexed, and its robustness. Confocal micro-Raman spectroscopy is rapid, on-site, and specific and only requires a minimal sample size, which can be used as a detection tool in blood and other biological matrices [26]. In comparison with other diagnostic technologies [27, 28], it can measure molecular vibrations and provide fingerprint signatures of various tissue biomolecules. As this technique is free from water interference, it can also be applied to diagnostic studies in vivo. In this study, we utilized confocal micro-Raman spectroscopy to identify molecular changes in irradiated $\mathrm{Hb}$ and to establish the relationship between micro-Raman spectra and hypertension LED-mediated therapy.

\section{Materials and Methods}

2.1. Sample Preparation. Following standard protocols, fresh peripheral venous blood $(5 \mathrm{~mL})$ was obtained by venipuncture from a patient with hypertension CVD and placed in glass tubes that contained acid citrate dextrose as an anticoagulant. Blood samples were stored in an $8^{\circ} \mathrm{C}$ refrigerator immediately after collection until they were required
TABLE 1: The detailed specification and parameters of red LED.

(a) Electrical-Optical Characteristics at $I_{F}=400 \mathrm{~mA}, \mathrm{Ta}=25^{\circ} \mathrm{C}$

\begin{tabular}{lccccc}
\hline Parameter & Symbol & Min & Type & Max & Unit \\
\hline Luminous & $\Phi_{v}$ & 30 & $\sim$ & 40 & $\mathrm{~lm}$ \\
Wavelength & $\lambda_{D}$ & 640 & $\sim$ & 660 & $\mathrm{~nm}$ \\
Forward voltage & $V_{F}$ & 2.0 & $\sim$ & 2.6 & $\mathrm{~V}$ \\
Power dissipation & $P_{D}$ & 0.80 & $\sim$ & 1.04 & $\mathrm{~W}$ \\
View angle & $2 \theta_{1 / 2}$ & $\sim$ & 60 & $\sim$ & $\mathrm{deg}$. \\
Thermal resistance & $R \theta_{J-B}$ & $\sim$ & 12 & $\sim$ & ${ }^{\circ} \mathrm{C} / \mathrm{W}$ \\
\hline
\end{tabular}

(b) Absolute Maximum Ratings

\begin{tabular}{lccc}
\hline Parameter & Symbol & Value & Unit \\
\hline Forward Current & $I_{F}$ & 400 & $\mathrm{~mA}$ \\
Junction Temperature & $T_{j}$ & 115 & ${ }^{\circ} \mathrm{C}$ \\
Operating Temperature & $T_{\text {opr }}$ & $-40 \sim+60$ & ${ }^{\circ} \mathrm{C}$ \\
Storage Temperature & $T_{\text {stg }}$ & $0 \sim+60$ & ${ }^{\circ} \mathrm{C}$ \\
ESD sensitivity & $\sim$ & $\pm 2,000 \mathrm{~V}$ & $\sim$ \\
Temperature Coefficient of voltage & $\sim$ & -5 & $\mathrm{Hv} /{ }^{\circ} \mathrm{C}$ \\
DC Pulse Current (@ 1 KHz, 10\% duty & $I_{\mathrm{FP}}$ & 1000 & $\mathrm{~mA}$ \\
cycle) & & \multicolumn{2}{c}{ Not designed for } \\
Reverse Voltage & $V_{R}$ & reverse operation \\
\hline
\end{tabular}

*Notes

1. Tolerance of Luminous Flux is $\pm 3 \%$.

2. Tolerance of Forward Voltage is $\pm 0.1 \mathrm{~V}$.

for irradiation or clinical measurements. The blood was centrifuged $\left(3000 \mathrm{rpm}, 5 \mathrm{~min}, 4^{\circ} \mathrm{C}\right)$ to produce a buffy coat. The erythrocytes were then harvested from the base of the tube and washed three times with isotonic phosphate buffered saline (PBS) at $\mathrm{pH} 7.4$.

The erythrocytes were placed in a culture dish with a diameter of $3 \mathrm{~cm}$, wherein the depth of the erythrocyte specimens was about $2 \mathrm{~mm}$. The distance between the LED lamp and the specimens was adjusted to $2 \mathrm{~cm}$, and the LED were applied vertically with a continuous power output of $15.0 \mathrm{~mW}$ (irradiance: $2.1 \mathrm{~mW} / \mathrm{cm}^{2}$ ) based on a previously published study [17]. The central wavelength of red LED was 620$640 \mathrm{~nm}$, which was comparable to the He-Ne laser device with a wavelength of $632.8 \mathrm{~nm}$ [21]. Different irradiation times were obtained by setting the timer to automatically turn off the power. The samples were irradiated for 5, 10, and $30 \mathrm{~min}$, respectively. The schematic drawing of red LED irradiation is shown in Figure 1. This irradiation device is made up of the control unit and array LED. The control unit could adjust the irradiation power and time. And the array LED is made up of 10 red LED beads (ShenZhen GeTian Optoelectronics Co., LTD, China) which are of super high flux output, high luminance, and low thermal resistance. The detailed specification and parameters of LED are depicted in Table 1. Afterwards, the isolated $\mathrm{Hb}$ was extracted from the irradiated erythrocytes.

In order to eliminate the impact of the erythrocyte membrane and investigate the effect of LED irradiation on $\mathrm{Hb}$ 


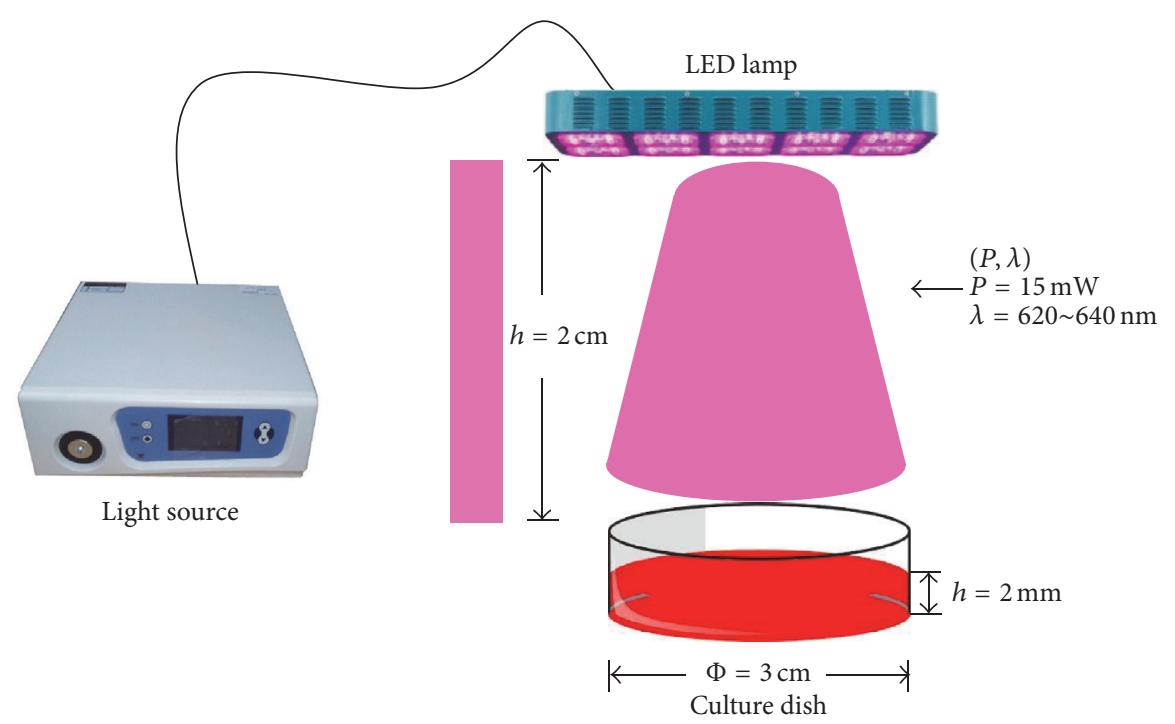

Figure 1: Schematic drawing of red light-emitting diode irradiation.

directly, the isolated $\mathrm{Hb}$ specimens were prepared by adding the irradiated erythrocytes to double distilled water at a volumetric ratio of $1: 1$. Next, the hemolytic erythrocytes were centrifuged at $3000 \mathrm{rpm}$ for $30 \mathrm{~min}$ at $4^{\circ} \mathrm{C}$. The supernatants were carefully separated and used as $\mathrm{Hb}$ samples, which were then placed in glass capillary tubes with a diameter of approximately $1 \mathrm{~mm}$ for Raman detection. We obtained the full local approval from the ethics committee of the university for all of the experiments described above.

2.2. Raman Microspectrometer. A Renishaw inVia confocal micro-Raman microscope was equipped with an $\times 50$ objective lens $(\mathrm{NA}=0.75)$ and a laser excitation of $514 \mathrm{~nm}$ was used to obtain all spectra. The resolution of this instrument was $1 \mathrm{~cm}^{-1}$ and the laser power that was focused on the samples was $0.15 \mathrm{~mW}$. Each spectrum was recorded with a $3 \mathrm{~s}$ exposure time and five accumulations. In our experiment, the $514 \mathrm{~nm}$ diode laser with a power of $0.15 \mathrm{~mW}$ had minimal effects of thermal degradation and photo degradation. The back-scattered Raman light with a recorded range of 600$1800 \mathrm{~cm}^{-1}$ was collected with a slit-width in our experiment of approximately $50 \mu \mathrm{m}$. Prior to initiating Raman scanning, the silicon wafer with a $520 \mathrm{~cm}^{-1}$ band was used for frequency calibration. All of the data were collected under the same conditions.

2.3. Atomic Force Microscopy (AFM). All erythrocyte samples were deposited on freshly cleaved mica surfaces and fixed with $2.5 \%$ glutaraldehyde for $5 \mathrm{~min}$. The fixed cells were washed five times with distilled water in order to avoid the disturbance of salinity and then air-dried at room temperature. AFM (AutoProbe CP, Veeco Instruments, Santa Barbara, USA) test was carried out in air and imaged in contact mode. Microfabricated silicon cantilevers with a constant force of approximately $2.8 \mathrm{~N} / \mathrm{m}$ were used. AFM images were planar leveled using Proscan Image Processing Software Version 2.1 (Thermo Microscopes) provided with the instrument in order to eliminate low frequency background noise in the scanning direction.

\section{Data Analysis}

In order to conduct statistical analysis, at least 45 Raman spectra were randomly obtained from each $\mathrm{Hb}$ sample. The final Raman spectrum was taken as the baseline that was corrected by the software program R 2.8.1 provided by Renishaw. Afterwards, the data was smoothed, normalized, and averaged by ORIGIN PRO 8.0 (Origin Lab Corporation, Northampton, MA, USA) [29, 30].

Principal component analysis (PCA), which is a multivariate analysis, is developed in the Matlab platform (The Mathworks, Inc., Natick, MA, USA) [31]. PCA is a technique of data dimensional reduction by orthogonally projecting data onto a lower dimensional linear space such that the variances of the projected data are maximized. It is an efficient approach for data classification and statistical data analysis. In our study, PCA was utilized to retain the most important information for $\mathrm{Hb}$ diagnosis and characterization. The analysis was oriented towards modeling a variance-covariance structure of a data matrix from which the eigen values that corresponded to principal components were extracted. Each principal component (PC) was a linear combination of the $n$ independent wave number variables $x_{1}, x_{2}, x_{3}, \ldots, x_{n}$. For example,

$$
\mathrm{PC} 1=\alpha_{1} x_{1}+\alpha_{2} x_{2}+\cdots+\alpha_{n} x_{n}
$$

The first PC accounts for the greatest variance and thus corresponds to the largest eigen value. The second $\mathrm{PC}$ is orthogonal to the first PC, with each successive PC being orthogonal to all those preceding it, and this accounts for a decreasing proportion of the variance. In this paper, we chose to analyze the first three PC items. 


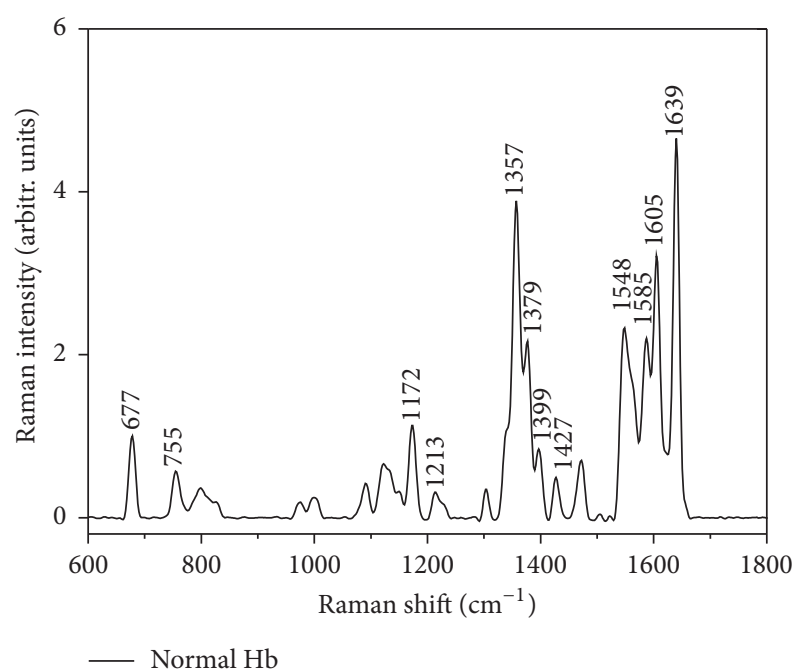

FIgURE 2: Averaged Raman spectra of normal hemoglobin from healthy person.

TABLE 2: Exquisite assignment of normal hemoglobin Raman bands from healthy person.

\begin{tabular}{lccc}
\hline Frequency $\left(\mathrm{cm}^{-1}\right)$ & Mode & Local coordinate & Symmetry \\
\hline 677 & $v_{7}$ & $\delta$ (pyr deform $)_{\text {sym }}$ & $\mathrm{A}_{1 \mathrm{~g}}$ \\
755 & $v_{15}$ & $\nu($ pyr breathing $)$ & $\mathrm{B}_{1 \mathrm{~g}}$ \\
1001 & Phe & $\nu\left(\mathrm{C}_{\beta} \mathrm{C}_{1}\right)$ & \\
1120 & $v_{22}$ & $\delta\left(\mathrm{CH}_{2}\right)$ twisting, wagging & $\mathrm{A}_{2 \mathrm{~g}}$ \\
1172 & $v_{30}$ & $\nu(\text { pyr half-ring })_{\text {asym }}$ & $\mathrm{B}_{2 \mathrm{~g}}$ \\
1213 & $v_{5}+v_{18}$ & $\delta\left(\mathrm{C}_{m} \mathrm{H}\right)$ & $\mathrm{B}_{1 \mathrm{~g}}$ or $\mathrm{E}_{\mathrm{u}}$ \\
1226 & $v_{13}$ & $\delta\left(\mathrm{C}_{m} \mathrm{H}\right)$ & $\mathrm{B}_{1 \mathrm{~g}}$ \\
1303 & $v_{21}$ & $\delta_{\text {asym }}\left(\mathrm{C}_{m} \mathrm{H}\right)$ & $\mathrm{A}_{2 \mathrm{~g}}$ \\
1357 & $v_{4}$ & $v(\text { pyr half-ring })_{\text {sym }}$ & $\mathrm{A}_{1 \mathrm{~g}}$ \\
1376 & $v_{4}$ & $\nu(\text { pyr half-ring })_{\text {sym }}$ & $\mathrm{A}_{1 \mathrm{~g}}$ \\
1399 & $v_{20}$ & $v($ pyr quater-ring $)$ & $\mathrm{A}_{2 \mathrm{~g}}$ \\
1427 & $v_{28}$ & $v\left(\mathrm{C}_{\alpha} \mathrm{C}_{m}\right)_{\text {sym }}$ & $\mathrm{B}_{2 \mathrm{~g}}$ \\
1548 & $v_{11}$ & $\nu\left(\mathrm{C}_{\beta} \mathrm{C}_{\beta}\right)$ & $\mathrm{B}_{1 \mathrm{~g}}$ \\
1585 & $v_{37}$ & $\nu\left(\mathrm{C}_{\alpha} \mathrm{C}_{m}\right)_{\text {asym }}$ & $\mathrm{E}_{\mathrm{u}}$ \\
1605 & $v_{19}$ & $\nu\left(\mathrm{C}_{\alpha} \mathrm{C}_{m}\right)_{\text {asym }}$ & $\mathrm{A}_{2 \mathrm{~g}}$ \\
1639 & $v_{10}$ & $v\left(\mathrm{C}_{\alpha} \mathrm{C}_{m}\right)_{\text {asym }}$ & $\mathrm{B}_{1 \mathrm{~g}}$ \\
\hline
\end{tabular}

\section{Results}

4.1. Raman Spectra of Hb from Healthy Person. The averaged Raman spectra of $\mathrm{Hb}$ from healthy person are shown in Figure 2. The $v_{7}$ band at $677 \mathrm{~cm}^{-1}$ that is assigned to the (pyrrole) sym is used to normalize all Raman spectra because its intensity remains the same as that for $\mathrm{Hb}$ of healthy person. The assignments of all major Raman peaks and local coordinates for $\mathrm{Hb}$ from healthy person are depicted in Table 2.

The $810 \mathrm{~cm}^{-1}$ peak corresponds to the $\mathrm{C}_{m} \mathrm{H}$ out-of-plane deformation stretching mode, while the 1213 and $1226 \mathrm{~cm}^{-1}$ peak corresponds to the $\mathrm{C}_{m} \mathrm{H}$ in-plane deformation bending mode. The bands that are located between 1100 and $1400 \mathrm{~cm}^{-1}$

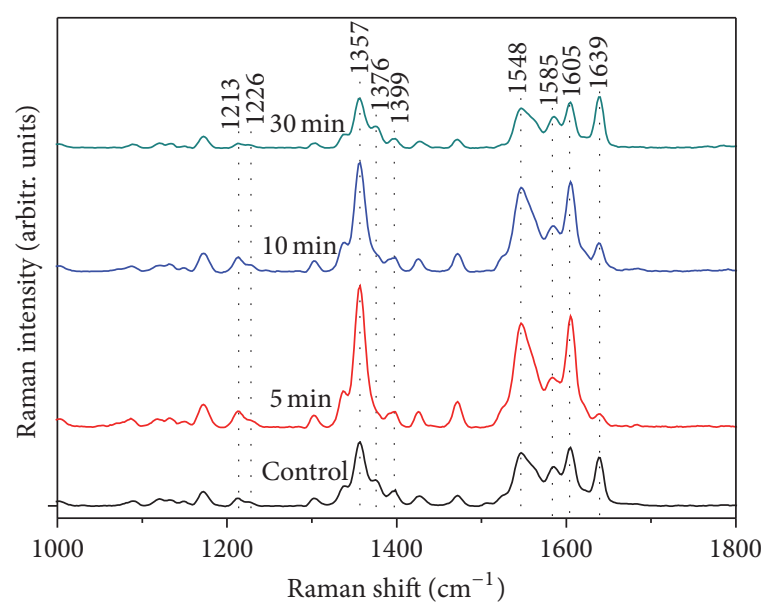

FIGURE 3: Raman spectra of controlled hemoglobin and irradiated hemoglobin from hypertension person.

attribute mainly to the pyrrole in-phase half-ring breathing vibrations. The band at $1226 \mathrm{~cm}^{-1}$ is the marker of oxygenated erythrocytes while the band at $1213 \mathrm{~cm}^{-1}$ is indicative of deoxygenated erythrocytes [32]. Also they are well-known markers for oxy-deoxy state of $\mathrm{Hb}$. It is assigned to $\nu_{5}+$ $\nu_{8}$ and $\nu_{13}$, respectively. The $1357 \mathrm{~cm}^{-1}$ peak is assigned to $v_{4}$. According to previously published studies $[33,34]$, the Raman peak at $1399 \mathrm{~cm}^{-1}\left(v_{20}\right)$ is the marker of heme aggregation. The $1585 \mathrm{~cm}^{-1}\left(\nu_{37}\right)$ peak corresponds to the $\mathrm{C}_{\alpha} \mathrm{C}_{m}$ asymmetric stretching mode. The core-size or spin state marker band region lies between 1500 and $1650 \mathrm{~cm}^{-1}$. In particular, the appearance of the $1639 \mathrm{~cm}^{-1}\left(\nu_{10}\right)$ band, which has the local coordinate character of $v\left(\mathrm{C}_{\alpha} \mathrm{C}_{m}\right)_{\text {asym }}$, is the characteristic of the $\mathrm{O}_{2}$ concentration marker in $\mathrm{Hb}$.

4.2. Raman Spectra of Controlled and Irradiated $\mathrm{Hb}$ from Hypertension Person. Figure 3 shows the spectra of controlled $\mathrm{Hb}(\mathrm{CH})$ and irradiated $\mathrm{Hb}(\mathrm{IH})$ in hypertension samples. Both the $\mathrm{CH}$ and $\mathrm{IH}$ Raman spectra indicated an extreme ordering of the hemes within cells. Figure 3 reveals that the band at 1213,1226 , and $1399 \mathrm{~cm}^{-1}$ decreased with prolonged irradiation time. The quantitative analysis of $1399 \mathrm{~cm}^{-1}$ peak is shown in Figure 4 . The intensities of the 1213,1226 , and $1399 \mathrm{~cm}^{-1}$ peaks decreased slightly in the first $10 \mathrm{~min}$; then an obvious decline was observed after $30 \mathrm{~min}$ of irradiation. Since the 1226 and $1213 \mathrm{~cm}^{-1}$ bands are the markers for oxy-deoxy state of $\mathrm{Hb}$, the decreased intensity of $1226 \mathrm{~cm}^{-1}$ and the comparable intensity of $1213 \mathrm{~cm}^{-1}$ compared to controlled $\mathrm{Hb}$ in our study perfectly presented the transition from oxygenated to deoxygenated state. Also the Raman peak at $1399 \mathrm{~cm}^{-1}$ is the marker of heme aggregation; the decreased intensity of $1399 \mathrm{~cm}^{-1}$ in our study indicated that heme aggregation was inhibited by prolonging the irradiation time. Obvious enhancements of intensity in the Raman bands at 1357, 1548, and $1605 \mathrm{~cm}^{-1}$ were observed after 5 min of irradiation. However, the intensity of bands at 1376,1585 , and $1639 \mathrm{~cm}^{-1}$ decreased conversely. According 


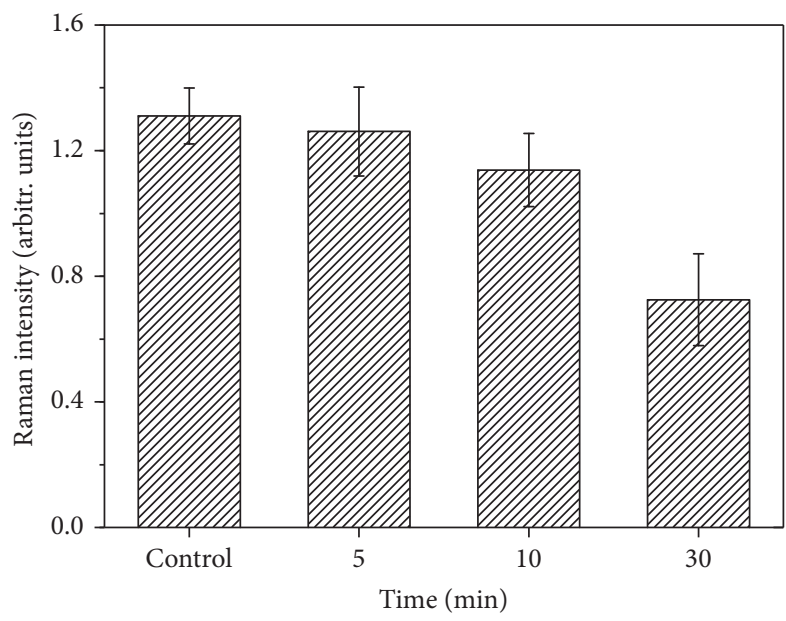

FIGURE 4: Quantitative analysis of Raman band $\left(1399 \mathrm{~cm}^{-1}\right)$ on irradiated hemoglobin from hypertension person.

to previous reports, bands at 1357,1548 , and $1605 \mathrm{~cm}^{-1}$ were enhanced in deoxygenated $\mathrm{Hb}$, while bands at 1376, 1585, and $1639 \mathrm{~cm}^{-1}$ increased in oxygenated $\mathrm{Hb}$ [35]. $1639 \mathrm{~cm}^{-1}$ is known as a symbolic band of $\mathrm{O}_{2}$ concentration. The decreased intensity of the $1639 \mathrm{~cm}^{-1}$ band indicated that the oxygenated $\mathrm{Hb}$ trend was not obvious in the first $5 \mathrm{~min}$ of light therapy.

When the irradiation time was extended to $30 \mathrm{~min}$, the intensities of the 1357,1548 , and $1605 \mathrm{~cm}^{-1}$ peaks were gradually decreased; however, the intensities of 1376, 1585, and $1639 \mathrm{~cm}^{-1}$ were gradually increased. The enhanced intensity seen at the $1639 \mathrm{~cm}^{-1}$ peak indicated that $\mathrm{Hb}$ could bind $\mathrm{O}_{2}$ more easily. Consequently, as with the marked peaks of oxygenated and deoxygenated $\mathrm{Hb}$ noted before [35], the oxygenated $\mathrm{Hb}$ content increased with prolongation of the irradiation time. However, after $10 \mathrm{~min}$ of irradiation, the intensity of the $1639 \mathrm{~cm}^{-1}$ band was still lower than that of the controlled band and exceeded the control level after $30 \mathrm{~min}$ of irradiation. The fluctuation of intensity at the $1639 \mathrm{~cm}^{-1}$ band indicated that the irradiated $\mathrm{Hb}$ had initially deoxygenated and then oxygenated during the overall irradiation process. This observation meant that a 30 min irradiation time with a power of $15.0 \mathrm{~mW}$ was suitable to inhibit heme aggregation and raising the oxygen-carrying ability of $\mathrm{Hb}$. The extracted $\mathrm{Hb}$ molecules from the erythrocytes could introduce artifacts for the measurement of their Raman spectra.

4.3. Principal Component Analysis. In this study, we chose the first three PC items to build a 3D-PCA [36]. The 3D plots of PC1, PC2, and PC3 that were calculated for $\mathrm{Hb}$ and irradiated for 5, 10, and $30 \mathrm{~min}$, respectively, are presented in Figure 5. A total of 45 Raman spectra from each sample were used for 3D-PCA. The plots showed that almost all Raman spectra of 5-IH were located to the upper left of the box, and the 10-IH spectra were located to the middle. By contrast, the $30-\mathrm{IH}$ spectra were located to the upper right of the box. This result shows that this was a good classification relative to other methods, and a one-to-one relationship was established for $\mathrm{Hb}$ in hypertension within different

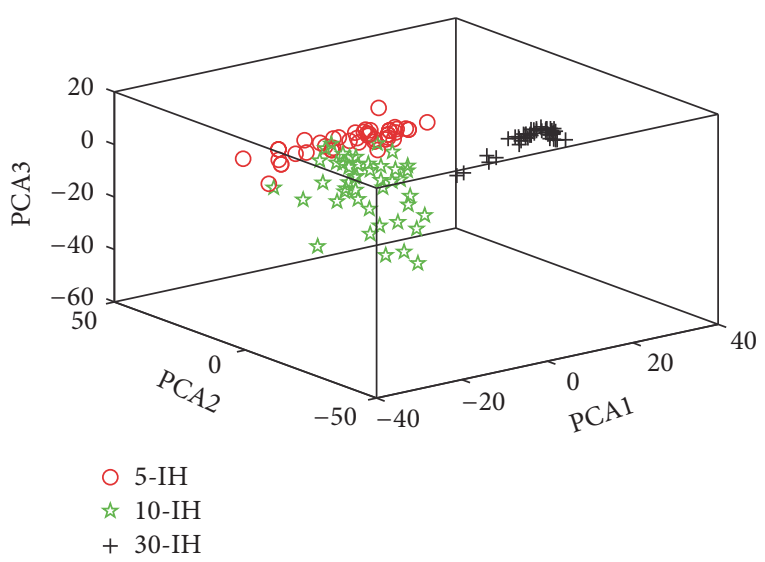

FIgure 5: First three PC (PC1, PC2, and PC3) scores plots of 3DPCA for irradiated hemoglobin from hypertension person $(5-\mathrm{IH}=$ irradiated $\mathrm{Hb}$ for $5 \mathrm{~min}, 10-\mathrm{IH}=$ irradiated $\mathrm{Hb}$ for $10 \mathrm{~min}$, and $30-\mathrm{IH}$ = irradiated $\mathrm{Hb}$ for $30 \mathrm{~min}$ ).

irradiation times and the corresponding Raman spectra. The excellent classification outcome seen in the 3D-PCA figure indicated that confocal micro-Raman spectroscopy provided a highly satisfactory approach to understand the molecular variations of irradiated $\mathrm{Hb}$ in hypertension. Confocal microRaman spectroscopy could be used to analyze the effects of hypertension treatment.

\section{Discussion}

Raman spectroscopic analysis is particularly attractive for biomedical purposes, especially given that it is an ideal tool in the detection and monitoring heme groups within single cells. In this study, we evaluated Raman spectral properties of $\mathrm{IH}$ with confocal Raman spectroscopy. The quantitative analysis depicted that the heme aggregation state was inhibited in $\mathrm{IH}$ spectra.

$\mathrm{Hb}$ catalyzes a variety of vital redox reactions in biological systems. It is largely dependent on the central iron- (Fe) containing moiety, which could take on various types of oxidation and spin states [37]. The Hb consists of a porphyrin macrocycle with an extended $\pi$ configuration. This porphyrin surrounds an Fe atom that is coordinated with four nitrogen atoms. In the deoxygenated state, the Fe atom is in the ferrous high spin state and the ferrous ion is combined with an $\mathrm{H}_{2} \mathrm{O}$ molecule below the porphyrin plane. While in the oxygenated state, the $\mathrm{Fe}$ atom is in a ferrous low spin state, and $\mathrm{O}_{2}$ will replace the $\mathrm{H}_{2} \mathrm{O}$ molecule. The changes in the electronic state are followed for $\mathrm{Hb}$ samples drawn at specific times during in vitro irradiation. Erythrocytes rely on the ferro-Hb to transport $\mathrm{O}_{2}$. However, metHb is in the ferric high spin state and thus cannot bind $\mathrm{O}_{2}$. In some cases, if the ferro- $\mathrm{Hb}$ are oxidized to metHb, they will lose the ability to transport oxygen. In this study, the oxygen marker band at $1639 \mathrm{~cm}^{-1}$ was increased obviously after $30 \mathrm{~min}$ LED irradiation. It indicated that the content of ferro-Hb was increased and oxygentransporting ability of $\mathrm{Hb}$ was promoted after irradiation. The results of in vitro erythrocytes experiment confirmed that 


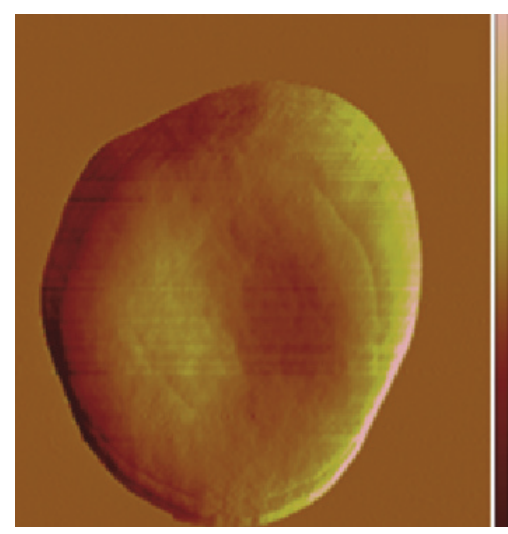

(a)

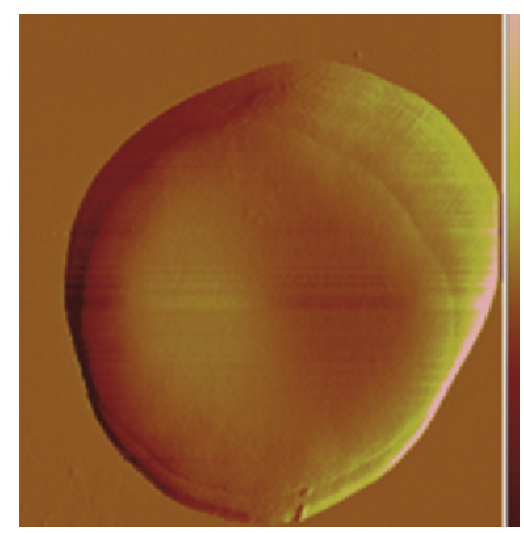

(b)

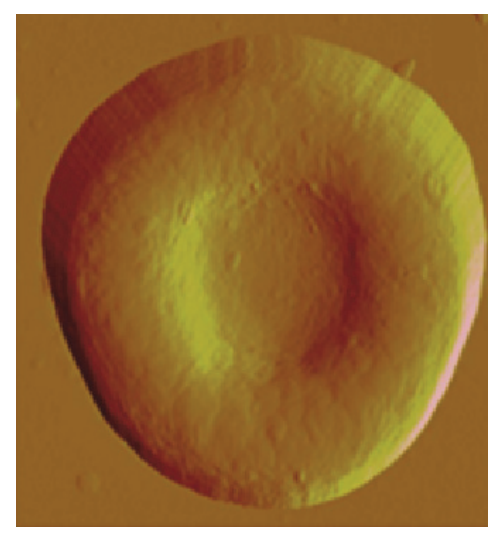

(c)

FIGURE 6: AFM data of the erythrocytes ((a) erythrocyte from hypertension person after irradiation, (b) erythrocyte from hypertension person before irradiation, and (c) erythrocyte from healthy person).

suitable LED irradiation conditions could alter the glycolysis of erythrocytes and the formation of ATP [38, 39], then preventing ferro- $\mathrm{Hb}$ from further oxidizing with metHb. Thus, LED irradiation could enhance the oxygen-carrying ability of erythrocytes and could be used to treat hypertension efficiently.

There are two main conformational states in $\mathrm{Hb}$. Raman spectroscopy has already been shown to be a powerful technique for monitoring the molecular dynamics of the R-form (i.e., relaxed conformation with a higher $\mathrm{O}_{2}$ concentration) to the T-form (i.e., tense conformation with a lower $\mathrm{O}_{2}$ concentration) of $\mathrm{Hb}$ [40]. It was shown that the reversible photodissociation of $\mathrm{Hb}$-ligand complexes, initiated by erythrocyte irradiation changed the oxyHb and deoxyHb levels, and the conformational transitions in the $\mathrm{Hb}$ macromolecules accompanying ligand detachment and addition were responsible for changes in their Raman spectra.

Figure 4 shows that the intensity of the $1399 \mathrm{~cm}^{-1}$ peak had decreased with the extension of the irradiation time, which is the heme aggregation marker. The decrease in intensity is primarily due to the excitonic interactions of the heme groups. The decreased peak value at the $1399 \mathrm{~cm}^{-1}$ band indicated that intermolecular distances of the heme groups dramatically increased during light therapy, which facilitated energy migration in the form of electronic transitions on the porphyrin network. The intensity of the $\mathrm{O}_{2}$ binding marker band at $1639 \mathrm{~cm}^{-1}$ had decreased in the first $10 \mathrm{~min}$, which was the $\mathrm{O}_{2}$ binding marker. LED irradiation led to the R-to-T state transition in irradiated $\mathrm{Hb}$. During this short duration, the $\mathrm{O}_{2}$-binding ability of $\mathrm{Hb}$ was not enhanced. When the irradiation time was prolonged from 5 to $30 \mathrm{~min}$, the intensity of the $1639 \mathrm{~cm}^{-1}$ band was gradually increased, implying that the T-to- $\mathrm{R}$ state transition of irradiated $\mathrm{Hb}$ occurred. The prolongation of the irradiation time (from 5 to $30 \mathrm{~min}$ ) led to an increase in the oxygenated $\mathrm{Hb}$ content during this period. Abundant LED irradiation promoted $\mathrm{Hb}$ to combine with $\mathrm{O}_{2}$ with the prolonged irradiation time. The increased peak intensity at marker bands of oxygenated $\mathrm{Hb}$ showed that $\mathrm{O}_{2}$ replaced $\mathrm{H}_{2} \mathrm{O}$ after a prolonged irradiation time, meaning that irradiation promoted the oxygen-transporting ability of $\mathrm{Hb}$. Previous studies also found that it could lead to an increase in the velocity of blood microcirculation [41]. The decrease of the band at $1399 \mathrm{~cm}^{-1}$ after 30 min of irradiation is that for this prolonged irradiation period the light first unfolds the deoxy $\mathrm{Hb}$ and then photodamages the formed heme aggregation.

In Figure 6, a low magnification $\left(10 \times 10 \mu \mathrm{m}^{2}\right.$ square scanning) view of both untreated and irradiated whole erythrocytes showed an image of a disk with a central depression and the diameters of the two kinds of cell were both approximately $8 \mu \mathrm{m}$. However, AFM image details indicated that there are some differences between the erythrocyte of hypertension after irradiation (a) and erythrocyte of hypertension (b). And the erythrocyte of hypertension after irradiation (a) looks close to normal person erythrocyte (c) in terms of the morphology. Figure 7 showed that the LED irradiation treated hypertension erythrocytes $(30 \mathrm{~min}$ ) did not show significant cellular morphology change/damage after 48 hours of culture. Therefore, this strategy could be considered as a safe method.

\section{Conclusions}

In this study, the red light LED irradiation effect on erythrocyte and electronic-conformational interactions in $\mathrm{Hb}$ molecules has been studied experimentally. We studied the molecular changes of irradiated $\mathrm{Hb}$ isolated from patients with hypertension components. Results confirmed that (I) the $1399 \mathrm{~cm}^{-1}$ band, which is the marker for heme aggregation, decreased gradually with prolonged irradiation time. It offers evidence supporting heme aggregation in response to photoinduced denaturation of $\mathrm{Hb}$ and demonstrated that LED irradiation could inhibit heme aggregation. (II) $v_{10}$ band at $1639 \mathrm{~cm}^{-1}$ is known as a marker band for $\mathrm{O}_{2}$ concentration. During the period of LED irradiation, the extent of deoxygenation initially decreased and then rapidly increased with prolonged irradiation time. (III) The bands at 1357,1548 , and $1605 \mathrm{~cm}^{-1}$ are marker bands of deoxygenated 


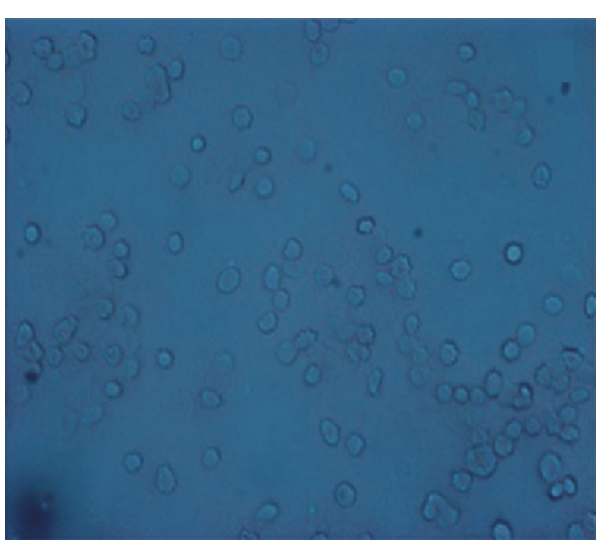

(a)

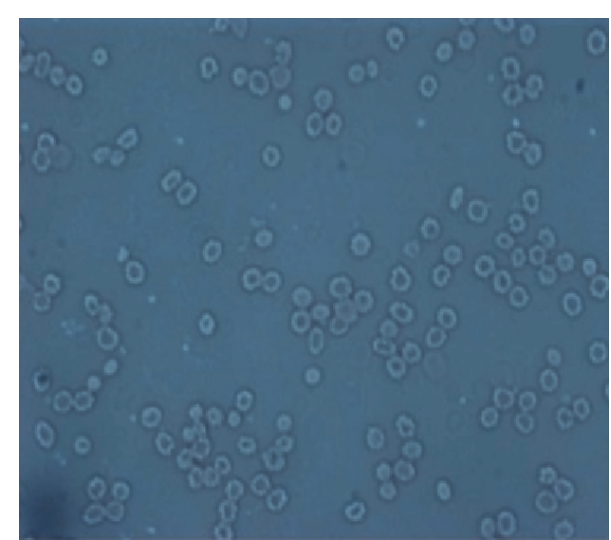

(b)

FIGURE 7: Microscopic images of red light irradiated and nonirradiated erythrocyte from hypertension person.

$\mathrm{Hb}$, while the bands at 1376,1585 , and $1639 \mathrm{~cm}^{-1}$ are the characteristic bands of oxygenated $\mathrm{Hb}$. The variation in the intensities indicates that, after $30 \mathrm{~min}$ of LED irradiation, the oxygenated $\mathrm{Hb}$ content would exceed the controlled $\mathrm{Hb}$ content during the light therapy process. (IV) The variance of intensities at the $1399 \mathrm{~cm}^{-1}$ and $1639 \mathrm{~cm}^{-1}$ bands showed that, in our study, the suitable LED irradiation time was $30 \mathrm{~min}$ with a power setting of $15.0 \mathrm{~mW}$. Experimental data showed that red LED phototherapy is feasible and effective at inhibiting heme aggregation and enhanced the $\mathrm{HbO}_{2}$ content in vitro. It provides persuasive experimental data in support of treating hypertension in vivo.

\section{Competing Interests}

The authors declare that they have no competing interests.

\section{Acknowledgments}

This work was supported by the National Natural Science Foundation of China (nos. 11404116, 61335011, 61275187, and 31300691), the Specialized Research Fund for the Doctoral Program of Higher Education of China (nos. 20134407120003 and 20114407110001), the PhD Startup Fund of Natural Science Foundation of Guangdong Province, China (no. S2013040016223), the Science and Technology Innovation Project of the Education Department of Guangdong Province of China (no. 2013KJCX0052), and the Young Teachers Nurturing Fund of South China Normal University (2012KJ020).

\section{References}

[1] P. M. Kearney, M. Whelton, K. Reynolds, P. Muntner, P. K. Whelton, and J. He, "Global burden of hypertension: analysis of worldwide data," The Lancet, vol. 365, no. 9455, pp. 217-223, 2005.

[2] Eurosurveillance Editorial Team, "WHO launches the World Health Statistics,” Eurosurveillance, vol. 17, no. 20, p. 15, 2012.
[3] J. R. Sowers, M. Epstein, and E. D. Frohlich, "Diabetes, hypertension, and cardiovascular disease: an update," Hypertension, vol. 37, no. 4, pp. 1053-1059, 2001.

[4] B. Dahlöf, R. B. Devereux, S. E. Kjeldsen et al., "Cardiovascular morbidity and mortality in the losartan intervention for endpoint reduction in hypertension study (LIFE): a randomised trial against atenolol," The Lancet, vol. 359, no. 9311, pp. 9951003, 2002.

[5] S. E. Kjeldsen, S. Julius, T. Hedner, and L. Hansson, "Stroke is more common than myocardial infarction in hypertension: analysis based on 11 major randomized intervention trials," Blood Pressure, vol. 10, no. 4, pp. 190-192, 2001.

[6] S. Laurent, S. Katsahian, C. Fassot et al., "Aortic stiffness is an independent predictor of fatal stroke in essential hypertension," Stroke, vol. 34, no. 5, pp. 1203-1206, 2003.

[7] R. Agarwal and M. J. Andersen, "Correlates of systolic hypertension in patients with chronic kidney disease," Hypertension, vol. 46, no. 3, pp. 514-520, 2005.

[8] P. A. Sarafidis, S. Li, S.-C. Chen et al., "Hypertension awareness, treatment, and control in chronic kidney disease," American Journal of Medicine, vol. 121, no. 4, pp. 332-340, 2008.

[9] V. Petkov, W. Mosgoeller, R. Ziesche et al., "Vasoactive intestinal peptide as a new drug for treatment of primary pulmonary hypertension," Journal of Clinical Investigation, vol. 111, no. 9, pp. 1339-1346, 2003.

[10] R. Seifie-Makrani, N. Sajjadi, O. Younesi, and H. Bagheri, "A new strategy for determination of captopril as a hypertension drug using zno nanoparticle modified carbon paste electrode," International Journal of Electrochemical Science, vol. 9, no. 4, pp. 1799-1811, 2014.

[11] G. Riegel, L. B. Moreira, S. C. Fuchs et al., "Long-term effectiveness of non-drug recommendations to treat hypertension in a clinical setting," American Journal of Hypertension, vol. 25, no. 11, pp. 1202-1208, 2012.

[12] G. S. Sainani, "Non-drug therapy in prevention and control of hypertension," Journal of Association of Physicians of India, vol. 51, pp. 1001-1006, 2003.

[13] R. P. Lifton, A. G. Gharavi, and D. S. Geller, "Molecular mechanisms of human hypertension," Cell, vol. 104, no. 4, pp. 545-556, 2001.

[14] Y. Shimizu, M. Nakazato, T. Sekita et al., "Association between the hemoglobin levels and hypertension in relation to the BMI 
status in a rural Japanese population: The Nagasaki Islands Study," Internal Medicine, vol. 53, no. 5, pp. 435-440, 2014.

[15] I. Anand, J. J. V. McMurray, M. Warren et al., "Anemia and its relationship to clinical outcome in heart failure," Circulation, vol. 110, no. 2, pp. 149-154, 2004.

[16] E. Lipšic, I. C. C. van der Horst, A. A. Voors et al., "Hemoglobin levels and 30-day mortality in patients after myocardial infarction," International Journal of Cardiology, vol. 100, no. 2, pp. 289-292, 2005.

[17] G.-Y. Luo, L. Sun, and T. C.-Y. Liu, "Aquaporin-1-mediated effects of low level He-Ne laser irradiation on human erythrocytes," International Journal of Photoenergy, vol. 2012, Article ID 275209, 5 pages, 2012.

[18] A. De Souza Da Fonseca, G. A. Presta, M. Geller, F. De Paoli, and S. S. Valença, "Low-intensity infrared laser increases plasma proteins and induces oxidative stress in vitro," Lasers in Medical Science, vol. 27, no. 1, pp. 211-217, 2012.

[19] G. M. Saied, R. M. Kamel, A. M. Labib, M. T. Said, and A. Z. Mohamed, "The diabetic foot and leg: Combined He-Ne and infrared low-intensity lasers improve skin blood perfusion and prevent potential complications. A prospective study on 30 Egyptian patients," Lasers in Medical Science, vol. 26, no. 5, pp. 627-632, 2011.

[20] S. C. Núñez, G. E. C. Nogueira, M. S. Ribeiro, A. S. Garcez, and J. L. Lage-Marques, "He-Ne laser effects on blood microcirculation during wound healing: a method of in vivo study through laser Doppler flowmetry," Lasers in Surgery and Medicine, vol. 35, no. 5, pp. 363-368, 2004.

[21] M. Gulsoy, G. H. Ozer, O. Bozkulak et al., "The biological effects of 632.8-nm low energy $\mathrm{He}-\mathrm{Ne}$ laser on peripheral blood mononuclear cells in vitro," Journal of Photochemistry and Photobiology B: Biology, vol. 82, no. 3, pp. 199-202, 2006.

[22] A. Tridente and D. De Luca, "Efficacy of light-emitting diode versus other light sources for treatment of neonatal hyperbilirubinemia: a systematic review and meta-analysis," Acta Paediatrica, vol. 101, no. 5, pp. 458-465, 2012.

[23] M. A. Naeser and M. R. Hamblin, "Potential for transcranial laser or LED therapy to treat stroke, traumatic brain injury, and neurodegenerative disease," Photomedicine and Laser Surgery, vol. 29, no. 7, pp. 443-446, 2011.

[24] D. Barolet, "Light-emitting diodes (LEDs) in dermatology," Seminars in Cutaneous Medicine and Surgery, vol. 27, no. 4, pp. 227-238, 2008.

[25] R. Weiss, D. McDaniel, R. Geronemus et al., "Clinical experience with Light-Emitting Diode (LED) photomodulation," Dermatologic Surgery, vol. 31, no. 3, supplement, pp. 1199-1205, 2005.

[26] P. Lemler, W. R. Premasiri, A. DelMonaco, and L. D. Ziegler, "NIR Raman spectra of whole human blood: effects of laserinduced and in vitro hemoglobin denaturation," Analytical and Bioanalytical Chemistry, vol. 406, no. 1, pp. 193-200, 2014.

[27] S. Zhuo, L. Zheng, J. Chen, S. Xie, X. Zhu, and X. Jiang, "Depthcumulated epithelial redox ratio and stromal collagen quantity as quantitative intrinsic indicators for differentiating normal, inflammatory, and dysplastic epithelial tissues," Applied Physics Letters, vol. 97, no. 17, Article ID 173701, 2010.

[28] S. Zhuo, J. Chen, G. Wu et al., "Quantitatively linking collagen alteration and epithelial tumor progression by second harmonic generation microscopy," Applied Physics Letters, vol. 96, no. 21, Article ID 213704, 2010.
[29] Z. Zhuang, N. Li, Z. Guo, M. Zhu, K. Xiong, and S. Chen, "Study of molecule variations in renal tumor based on confocal microRaman spectroscopy," Journal of Biomedical Optics, vol. 18, no. 3, Article ID 031103, 2013.

[30] N. Li, S. X. Li, Z. Y. Guo et al., "Micro-Raman spectroscopy study of the effect of Mid-Ultraviolet radiation on erythrocyte membrane," Journal of Photochemistry and Photobiology B: Biology, vol. 112, pp. 37-42, 2012.

[31] B. R. Wood, P. Caspers, G. J. Puppels, S. Pandiancherri, and D. McNaughton, "Resonance Raman spectroscopy of red blood cells using near-infrared laser excitation," Analytical and Bioanalytical Chemistry, vol. 387, no. 5, pp. 1691-1703, 2007.

[32] B. R. Wood, B. Tait, and D. McNaughton, "Micro-Raman characterisation of the $\mathrm{R}$ to $\mathrm{T}$ state transition of haemoglobin within a single living erythrocyte," Biochimica et Biophysica Acta-Molecular Cell Research, vol. 1539, no. 1-2, pp. 58-70, 2001.

[33] B. R. Wood, L. Hammer, L. Davis, and D. McNaughton, "Raman microspectroscopy and imaging provides insights into heme aggregation and denaturation within human erythrocytes," Journal of Biomedical Optics, vol. 10, no. 1, Article ID 014005, 13 pages, 2005.

[34] B. R. Wood, L. Hammer, and D. McNaughton, "Resonance Raman spectroscopy provides evidence of heme ordering within the functional erythrocyte," Vibrational Spectroscopy, vol. 38, no. 1-2, pp. 71-78, 2005.

[35] Y. H. Kim, D. H. Jeong, D. Kim et al., "Photophysical properties of long rodlike meso-meso-linked zinc(II) porphyrins investigated by. Time-resolved laser spectroscopic methods," Journal of the American Chemical Society, vol. 123, no. 1, pp. 76-86, 2001.

[36] Z. Zhuang, M. Zhu, Y. Huang et al., "Study of molecule variation in various grades of human nuclear cataracts by confocal microRaman spectroscopy," Applied Physics Letters, vol. 101, no. 17, Article ID 173701, 3 pages, 2012.

[37] M. Casella, A. Lucotti, M. Tommasini et al., "Raman and SERS recognition of $\beta$-carotene and haemoglobin fingerprints in human whole blood," Spectrochimica Acta-Part A: Molecular and Biomolecular Spectroscopy, vol. 79, no. 5, pp. 915-919, 2011.

[38] T. Karu, L. Pyatibrat, and G. Kalendo, "Irradiation with He-Ne laser increases ATP level in cells cultivated in vitro," Journal of Photochemistry and Photobiology, B: Biology, vol. 27, no. 3, pp. 219-223, 1995.

[39] G. M. Saied, R. M. Kamel, A. M. Labib, M. T. Said, and A. Z. Mohamed, "The diabetic foot and leg: combined He-Ne and infrared low-intensity lasers improve skin blood perfusion and prevent potential complications. A prospective study on 30 Egyptian patients," Lasers in Medical Science, vol. 26, no. 5, pp. 627-632, 2011.

[40] T. Spiro and X. Li, "Resonance Raman spectroscopy of metalloporphyrins," in Biological Applications of Raman Spectroscopy, T. G. Spiro, Ed., pp. 1-38, John Wiley \& Sons, New York, NY, USA, 1988

[41] A. N. Korolevich, N. S. Dubina, S. I. Vecherinskii, and M. S. Belsley, "Influence of low-intensity laser radiation on the rheological characteristics of human blood," Journal of Applied Spectroscopy, vol. 71, no. 4, pp. 572-579, 2004. 

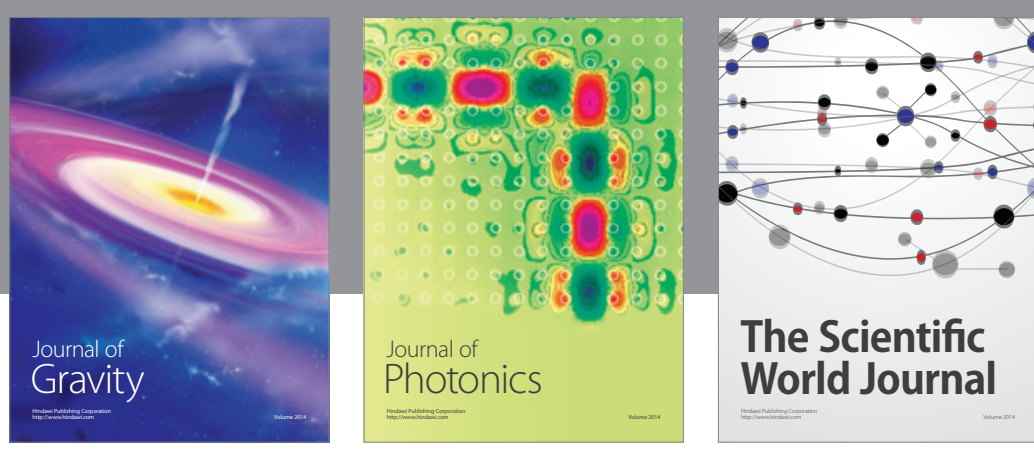

The Scientific World Journal
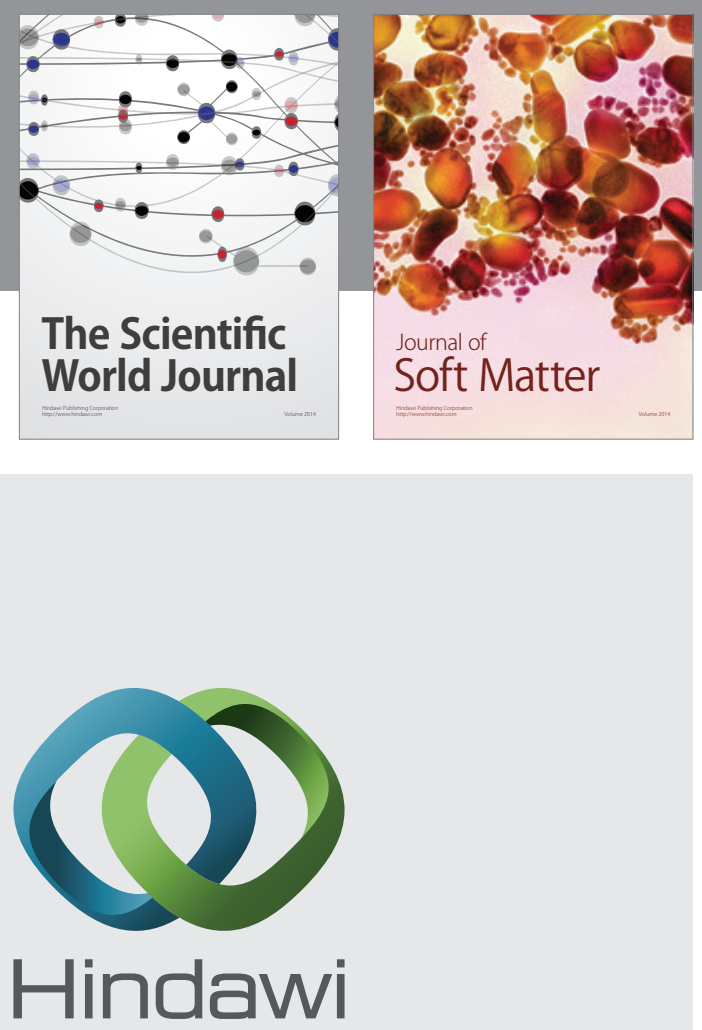

Submit your manuscripts at

https://www.hindawi.com
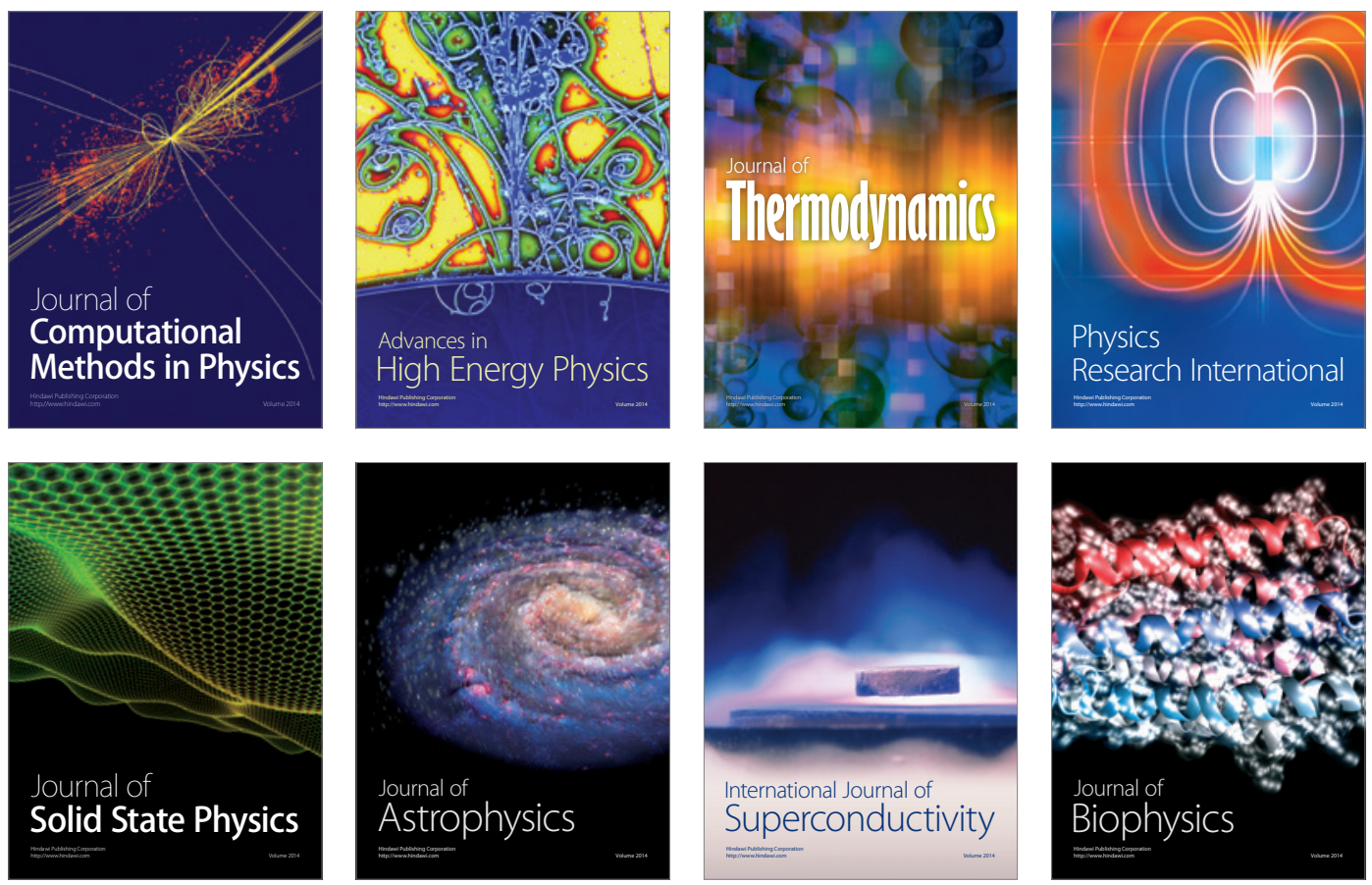
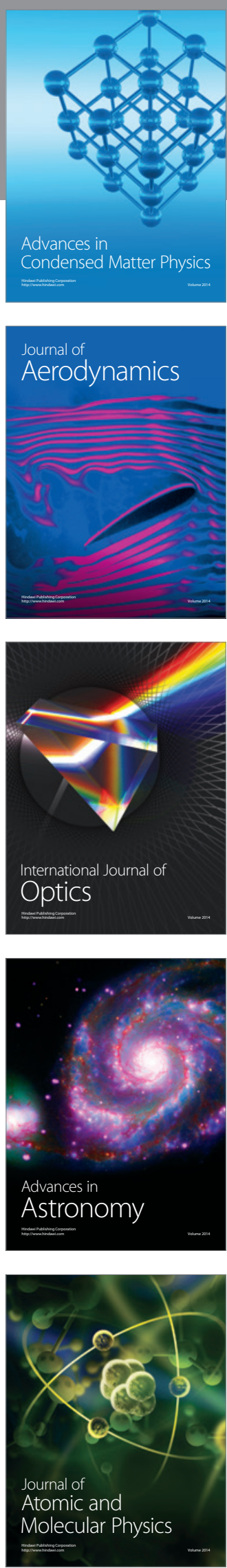